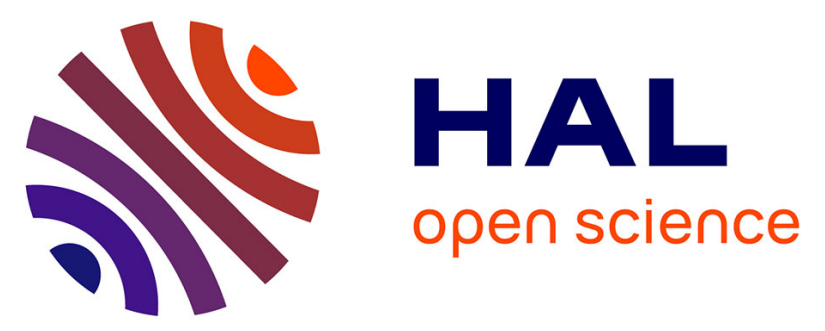

\title{
Accurate Prediction of the S 1 Excitation Energy in Solvated Azobenzene Derivatives via Embedded Orbital-Tuned Bethe-Salpeter Calculations
}

Aseem Rajan Kshirsagar, Gabriele D'avino, Xavier Blase, Jing Li, Roberta Poloni

\section{To cite this version:}

Aseem Rajan Kshirsagar, Gabriele D'avino, Xavier Blase, Jing Li, Roberta Poloni. Accurate Prediction of the S 1 Excitation Energy in Solvated Azobenzene Derivatives via Embedded Orbital-Tuned Bethe-Salpeter Calculations. Journal of Chemical Theory and Computation, 2020, 16 (4), pp.20212027. 10.1021/acs.jctc.9b01257 . hal-02503936

\section{HAL Id: hal-02503936 \\ https://hal.science/hal-02503936}

Submitted on 8 Dec 2020

HAL is a multi-disciplinary open access archive for the deposit and dissemination of scientific research documents, whether they are published or not. The documents may come from teaching and research institutions in France or abroad, or from public or private research centers.
L'archive ouverte pluridisciplinaire HAL, est destinée au dépôt et à la diffusion de documents scientifiques de niveau recherche, publiés ou non, émanant des établissements d'enseignement et de recherche français ou étrangers, des laboratoires publics ou privés. 


\section{Accurate prediction of the $S_{1}$ excitation energy in solvated azobenzene derivatives via embedded orbital-tuned Bethe-Salpeter calculations

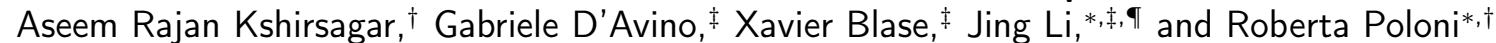 \\ $\dagger$ Grenoble-INP, SIMaP, University of Grenoble-Alpes, CNRS, F-38042 Grenoble, France \\ $\ddagger$ Institut Néel-CNRS and Université Grenoble-Alpes, F-38042 Grenoble, France \\ 【Université Grenoble-Alpes, CEA, IRIG, MEM-L_Sim, F-38000 Grenoble, France \\ Received March 5, 2020; E-mail: jing.li@cea.fr; roberta.poloni@grenoble-inp.fr}

\begin{abstract}
By employing the Bethe-Salpeter formalism coupled with a non-equilibrium embedding scheme, we demonstrate that the paradigmatic case of $\mathrm{S}_{1}$ band separation between cis and trans in azobenzene derivatives can be computed with excellent accuracy compared to experimental optical spectra. Besides embedding, we show that the choice of the Kohn-Sham exchange correlation functional for DFT is critical, despite the iterative convergence of $G W$ quasiparticle energies. We address this by adopting an orbital-tuning approach via the global hybrid functional, PBEh, yielding an environment-consistent ionization potential. The vertical excitation energy of 20 azo molecules are predicted with a mean absolute error as low as $0.06 \mathrm{eV}$, up to three times smaller compared to standard functionals such as M06-2X and PBE0, and five times smaller compared to recent TDDFT results.
\end{abstract}

In recent years, molecular photoswitches have attracted much attention from researchers in different disciplines since they offer an efficient way to achieve light-harvesting through molecular functionalization. Azobenzene (AB) and its derivatives constitute an important family of molecular photoswitches which has found a variety of applications such as in drug delivery, ${ }^{1,2}$ DNA and RNA manipulation, ${ }^{3,4}$ gas separation using metal-organic framework scaffolds, ${ }^{5-7}$ and many more.

The trans-cis isomerization in $\mathbf{A B}$ occurs upon UV or visible light irradiation. For device design, the yield of isomerization should be as high as possible to achieve the largest possible change in the physical properties of the system. ${ }^{5,6}$ An efficient way to achieve high fractions of cis or trans is to irradiate using wavelengths that give a selective absorption of one isomer over the other. ${ }^{8,9}$ In the case of $\mathbf{A B}$, only an incomplete yield of isomerization can be obtained upon $n \rightarrow \pi^{*}\left(\mathrm{~S}_{1}\right)$ excitation because of the $\mathrm{S}_{1}$ optical bands of cis and trans largely overlap. ${ }^{10}$ In this regard, azobenzene functionalization has been proposed as a successful way to separate the $\mathrm{S}_{1}$ between trans and cis and thus achieve almost complete fractions of each isomer at the photostationary state. ${ }^{11,12}$

In order to assist experimental efforts devoted to material design using azobenzene derivatives, and other photoactive molecules in general, computational methods yielding an accuracy of below a tenth of $\mathrm{eV}$ are required. In this Letter, we demonstrate that the Bethe-Salpeter equation (BSE) $/ G W$ formalism coupled with a non-equilibrium embedding scheme can yield excitation energies of the $\mathrm{S}_{1}$ in azobenzene derivatives in excellent agreement with experiments, yielding a mean absolute error as low as $0.06 \mathrm{eV}$ which is up to five times smaller compared to recent time- dependent density functional theory (TDDFT) calculations performed with embedding. ${ }^{13}$ Our approach is based on two main points. The first is that environment effects are taken into account both at the ground state level and the BSE/GW level by computing, respectively, the solvent-polarized KohnSham (KS) ground state and the reaction field describing the fast electronic response of the environment to excitations, employing here a discrete polarizable model. The second aspect addresses the large dependence found here of the computed optical spectra on the starting point functional. The DFT starting point is here chosen in accordance to the ionization potentialtheorem for KS DFT (i.e. $\epsilon_{H}=$-IP, with $\mathrm{IP}=$ ionization potential $)^{14-18}$ thus yielding a different starting point functional in gas phase than in solution.

The BSE/GW formalism, ${ }^{19}$ which was originally developed and then applied in the condensed matter community to describe optical excitations in semiconductors, ${ }^{19-21}$ has recently attracted attention even in the world of molecular systems, ${ }^{22-24}$ due to its accuracy and efficiency compared with wavefunction-based methods like CASPT2 $2^{25,26}$ or coupled-cluster, ${ }^{27}$ and its ability to overcome some of the limitations of TDDFT such as charge-transfer excitations and Rydberg excitations. ${ }^{22,28}$ Additionally, compared to TDDFT, the BSE/ $G W$ approach has two advantages, i) higher accuracy of $G W$ quasiparticle eigenvalues as compared to $\mathrm{KS}$ eigenvalues and ii) accurate asymptotic behavior of the two-particle (electron and hole) BSE Hamiltonian. $^{29}$ It is a two-step approach. First, within the $G W$ many-body perturbation theory, where $G$ is the timeordered single-particle Green's function and $W$ the screened Coulomb potential, the KS eigenvalues are corrected perturbatively yielding the true energies to add or remove one electron, also referred to as quasiparticle (QP) energies. The second step is to use BSE to describe a two-particle bound state, i.e. the excited electron and the hole that it leaves behind. Recently, the BSE/ $G W$ formalism has been further developed to incorporate the effects of classically described environments. $^{30-33}$

In this work, we use the BSE/ $G W$ as implemented in the FIESTA package, ${ }^{34,35}$ starting from the KS states $\left(\epsilon_{n}^{K S}, \phi_{n}^{K S}\right)$ obtained from a DFT calculation using the NWCHEM package. ${ }^{36}$ We perform eigenvalue self-consistent evGW calculations meaning that the $G W$ quasiparticle energy of $n$ level is updated iteratively without updating the KS wavefunction. ${ }^{35,37}$ Hence, the quasiparticle energy reads:

$$
\epsilon_{n}^{G W}=\epsilon_{n}^{K S}+\left\langle\phi_{n}^{K S}\left|\Sigma^{G W}\left(\epsilon_{n}^{G W}\right)-V^{X C}\right| \phi_{n}^{K S}\right\rangle,
$$

and it converges normally within a few iterations.

To compute accurately the optical spectra of solvated molecules, the solvation effects need to be taken into account since they are known to bring significant changes in 


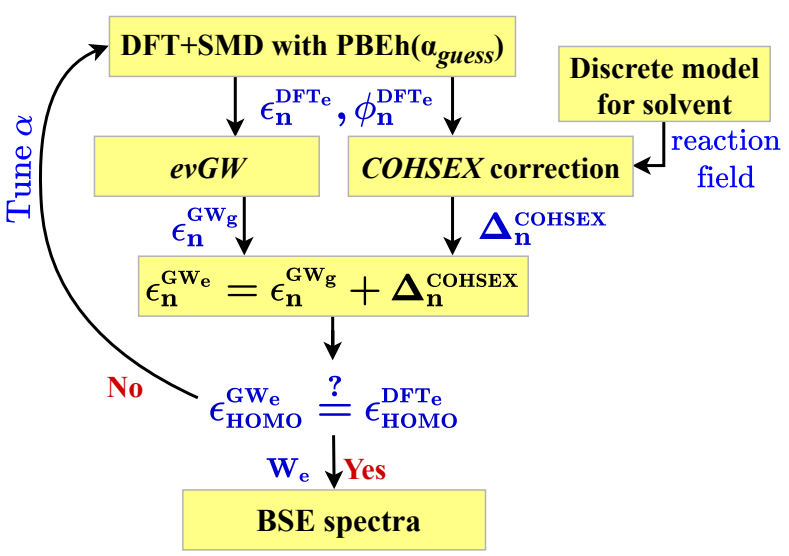

Figure 1. Workflow of the non-equilibrium embedded BSE $/ G W$ scheme adopted in this work.

the spectral features, as discussed for the case of azobenzene in the review work by Bandara and Burdette. ${ }^{10}$ Here, we employ a QM/MM (Quantum Mechanics/Molecular Mechanics) approach where the solvent is accounted for at both the DFT and BSE/GW level. To describe the ground state polarization effects, among available polarizable continuum models (PCM), we employ the solvation model based on the density $(\mathrm{SMD})^{38}$ whose parameters are taken from the Minnesota solvent descriptor database. ${ }^{39}$ At BSE/ $G W$ level, the fast electronic response of the MM part is described by the reaction field matrix calculated using the solvent optical dielectric constant, $\epsilon_{\text {opt }}$, which adds the contribution of the reaction field from the MM part to the screened Coulomb potential, $W^{31,32,40,41}$. Specifically, starting from the screened Coulomb potential, $W$, verifying the Dyson equation for the gas-phase molecule:

$W\left(r, r^{\prime} ; \omega\right)=v\left(r, r^{\prime}\right)+\int d r_{1} d r_{2} v\left(r, r_{1}\right) \chi_{0}\left(r_{1}, r_{2} ; \omega\right) W\left(r_{2}, r^{\prime} ; \omega\right)$

with $\chi_{0}$ being the independent-particle susceptibility, the solvent effects are incorporated by solving the following set of equations, written in compact notation:

$$
\begin{gathered}
\tilde{v}=v+v \chi^{M M} v \\
W_{e}=\tilde{v}+\tilde{v} \chi_{0} W_{e}
\end{gathered}
$$

where the screened Coulomb potential incorporating solvent effects is $W_{e}$ and the modified Coulomb potential, $\tilde{v}$, is the bare Coulomb potential, $v$, plus a renormalization term including the solvent reaction field. To avoid dealing with a full frequency-dependent $\chi^{M M}$, we follow Refs. ${ }^{40,41}$ and adopt a strategy that consists in merging the low-frequency optical limit of $G W$ with a discrete polarizable model that uses a low-frequency optical dielectric constant (e.g. $\epsilon_{\text {opt }}=1.77$ for methanol). The static Coulomb-hole plus screened exchange (COHSEX) approach is thus used to calculate $W_{e}$ and the difference between COHSEX self-energy calculated with and without solvent is added to the evGW eigenvalues to get the $\epsilon_{n}^{G W_{e}}$ :

$$
\begin{aligned}
\epsilon_{n}^{G W_{e}} & \approx \epsilon_{n}^{G W}+\Delta \epsilon_{n}^{\mathrm{COHSEX}} \\
& =\epsilon_{n}^{G W}+\left\langle\phi_{n}^{K S}\left|\Sigma^{\mathrm{COHSEX} / \mathrm{MM}}-\Sigma^{\mathrm{COHSEX}}\right| \phi_{n}^{K S}\right\rangle
\end{aligned}
$$

The $W_{e}$ is subsequently used for building the BSE hamiltonian. Fig. 1 illustrates the whole computational scheme.

This embedded many-body perturbation approach includes the environmental dielectric effect on both

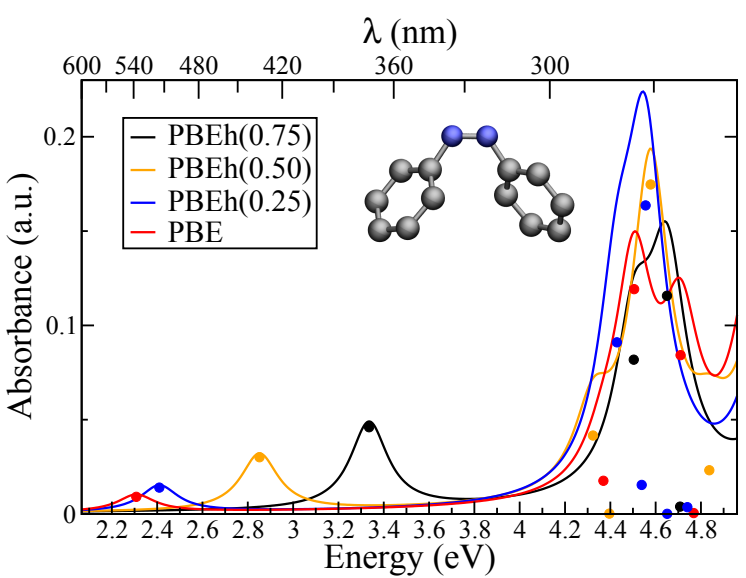

Figure 2. Optical spectra of cis-AB in gas phase calculated with $\mathrm{BSE} / G W / \mathrm{DFT} @ \operatorname{PBEh}(\alpha)$, with $\alpha$ being the fraction of exact exchange. The spectra show a large dependence on DFT functional. $\mathrm{E}\left(\mathrm{S}_{1}\right)$ increases by around $1 \mathrm{eV}$ when $\alpha$ is increased from 0 to 0.75 .

charged $^{31,40}$ and neutral excitations, ${ }^{41}$ and has demonstrated the accuracy of the order of a tenth of $\mathrm{eV}$ in the prediction of the ionization potential. ${ }^{32}$ Both linear response and state specific solvent contributions are accounted for in the present BSE scheme. ${ }^{41}$ In this work, the reaction field matrix is determined by a discrete polarizable model using the MESCAL code. ${ }^{42}$ The solvent is described by a cubic lattice of polarizable points with the polarizability determined by the Clausius-Mossotti relation. The convergence studies for the solvent model is reported in SI (Table S2 and S3) together with validation against literature data computed using the more standard PCM approach (Table $\mathrm{S} 4)$. The optical dielectric constant, $\epsilon_{\text {opt }}$, is computed from the index of refraction at optical frequencies tabulated in the Minnesota solvent descriptor database. ${ }^{43}$ To distinguish the embedded calculations from the gas phase, we use the symbol $G W_{e}$ in contrast to $G W_{g}$ and similar symbols are used for DFT calculations. DFT ${ }_{e}$ refers to DFT with SMD. We name the full embedding scheme BSE $/ G W_{e} / \mathrm{DFT}_{e}$. The spectra are shown by multiplying the oscillator strength by a Lorentzian of $0.1 \mathrm{eV}$ broadening (HWHM). Geometrical optimization of $\mathbf{A B}$ and its derivatives is computed with NWCHEM be employing cc-pVTZ/PBE0/SMD level ${ }^{38,44,45}$ while the aug-cc-pVDZ basis set ${ }^{46}$ is employed for the rest of the DFT calculations needed to generate the KS eigenstates for subsequent $G W$ and BSE calculations.

Because we employ the evGW approach, a dependence on the DFT starting point one-body wavefunctions may be expected. Therefore, the choice of the exchange and correlation functional (XCF) may become important. To illustrate this, we compare the BSE spectra of $\mathbf{A B}$ computed with different DFT starting points (see Fig. 2), using a hybrid functional, ${ }^{44,47} \operatorname{PBEh}(\alpha)$, by varying the amount of exact exchange $\alpha \cdot \mathrm{PBEh}^{44} \mathrm{XCF}$ is expressed as:

$$
E_{X C}^{P B E h}=\alpha E_{X}^{\text {exact }}+(1-\alpha) E_{X}^{P B E}+E_{C}^{P B E}, \alpha \in[0,1] .
$$

where $E_{X}^{\text {exact }}$ is the exact exchange, $E_{X}^{P B E}$ and $E_{C}^{P B E}$ are PBE exchange and correlation, ${ }^{48}$ respectively. Fig. 2 demonstrates a notable change on both the excitation energy and the oscillator strength as a function of the XCF. Specifically, the first excitation energy of cis- $\mathbf{A B}, \mathrm{E}\left(\mathrm{S}_{1}\right)$, changes by as much as $\sim 1 \mathrm{eV}$ from $\alpha$ equal to 0 (i.e. PBE) to 0.75 , while the oscillator strength changes from 0.009 to 0.044 . In agreement with previous works, ${ }^{22,49}$ we observe a larger 
dependence of $\mathrm{S}_{1}$, exhibiting a $n \rightarrow \pi^{*}$ character, than $\mathrm{S}_{2}$ (with $\pi \rightarrow \pi^{*}$ character) on the XCF choice. A similar dependence is found for AB-trans (see Fig. S1). Such a large discrepancy on optical spectra can be attributed to significant changes in the input KS wavefunctions from one functional to another (see Fig. S3 and S4). The same qualitative result is obtained when comparing different functionals within the M06 series $^{50}$ (see Fig. S2 in SI).

To avoid such impasse, we choose a starting XCF that fulfills the ionization potential theorem. ${ }^{14-18}$ Practically, we choose a value of $\alpha$ that minimizes the following quantity,

$$
J(\alpha)=\left|\epsilon_{H}^{e v G W}(\alpha)-\epsilon_{H}^{K S}(\alpha)\right|,
$$

which is the difference between the HOMO energies in evGW and DFT. The IP is approximated here by the quasiparticle energy, evGW, computed at each value of $\alpha$. This approach allows us to resolve the arbitrariness in the choice of the starting XCF while improving the asymptotic decay of the density. Because the highest occupied KS orbital dominates the asymptotic decay of the density ${ }^{51-53}\left(\phi_{H}^{K S}(r) \rightarrow\right.$ $e^{-\sqrt{-2 \epsilon_{H}}|r|}$, and because the exact density of a N-electron system exponentially decays as ${ }^{54} n(r) \rightarrow e^{-2 \sqrt{-2 I P}|r|}$, the orbital tuning performed via minimization of equation 7 yields an asymptotic behavior of the density close to the exact-density case.

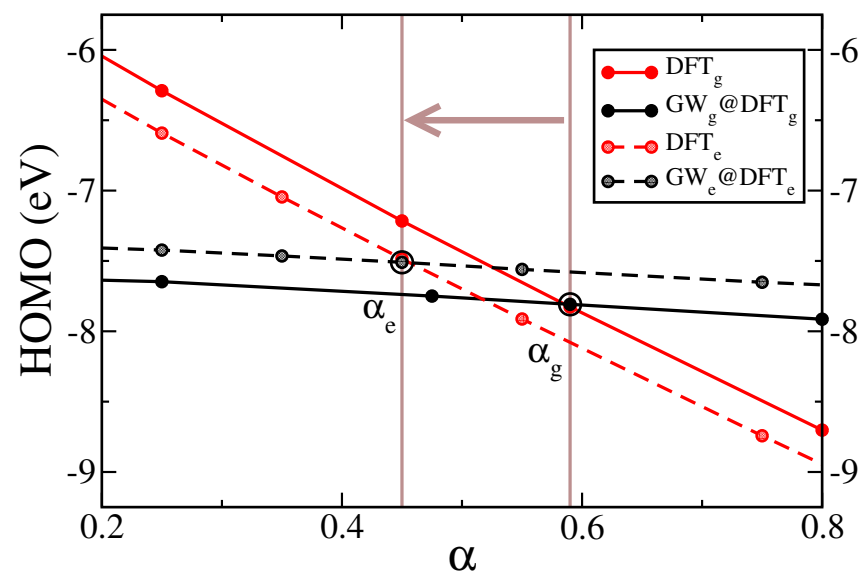

Figure 3. HOMO energy of AB-cis in gas and methanolsolvated phase as a function of $\alpha$ showing the values of $\alpha$ that minimize $\mathrm{J}(\alpha)$ for gas phase and embedding. The decrease in IP due to environmental screening effects reduces $\alpha$ from 0.59 to 0.45 .

In Fig. 3, we show $\epsilon_{H}^{K S}$ and $\epsilon_{H}^{G W}$ computed as a function of $\alpha$ for gas phase AB-cis and see that the condition that minimizes $\mathrm{J}(\alpha)$ is satisfied for $\alpha_{g}=0.59$, where ' $\mathrm{g}$ ' subscript, we remind, stands for gas phase. We compare the $\mathrm{S}_{1}$ and $\mathrm{S}_{2}$ excitation energies and oscillator strengths of cis and trans $\mathbf{A B}$ computed with this approach with recent coupled cluster calculations ${ }^{27}$ performed with aug-ccpVTZ basis set, and find an excellent agreement with our $\mathrm{BSE} / \mathrm{GW}_{g} / \mathrm{DFT}_{g}$ results. The comparison of the excitation energies is reported in Table S5, together with results from previous CASPT2 ${ }^{55}$ and recent TDDFT $^{13}$ calculations.

The inclusion of solvent polarization at $G W_{e}$ level narrows the electronic gap of cis and trans $\mathbf{A B}$ (see Fig. S5): the electron removal energies decrease as shown in Fig. 3 whereas the electron addition energies increase due to the dielectric screening of the hole and electron. This polarization effect results in a smaller value of $\alpha$ arising from the matching of $\epsilon^{G W_{e}}$ and $\epsilon^{K S, D F T_{e}}$, i.e. $\alpha_{e}=0.45$. This lower-

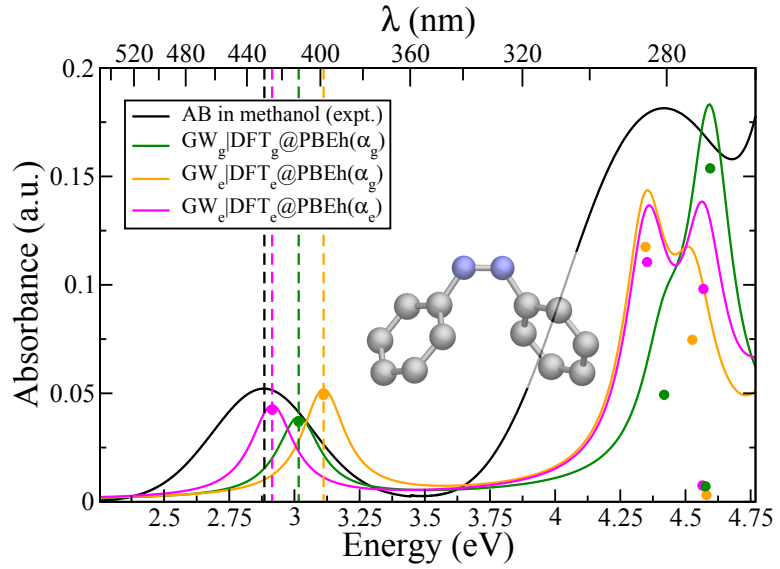

(a) 1-cis

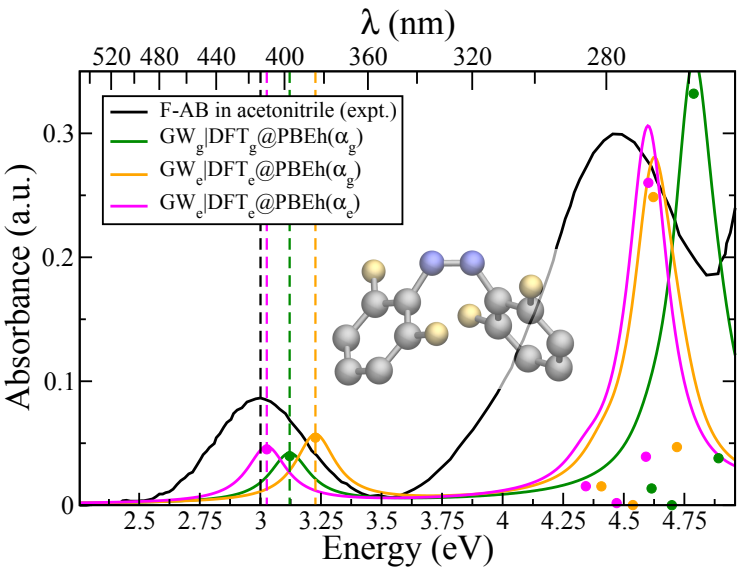

(b) 2-cis

Figure 4. BSE optical spectra of 1-cis and 2-cis calculated with different embedding schemes. For both molecules, a good agreement with experiment is obtained when the starting XCF is chosen using the environment consistent apprach described in the text. Experimental spectra in acetonitrile are in black ${ }^{8,12}$ and are arbitrarily scaled for better comparison.

ing of $\alpha$ is thus needed within DFT to follow the decrease in IP and thus mimics the screening of the charged excitation by the environment. We note here that the inclusion of the ground state polarization using the SMD model, i.e. $\mathrm{DFT}_{e}$, lowers the KS eigenvalues by $0.3 \mathrm{eV}$ and the quasiparticle energies $G W_{g} / \mathrm{DFT}_{e}$ by the same amount (i.e. without embedding at $G W)$. Thus, the dynamical screening by the solvent needs to be accounted for at the $G W_{e}$ level so as to determine an environment consistent IP. There has been much effort in the past few years in combining optimal tuning of range-separated hybrid functionals (OT-RSH) with classical solvation models. ${ }^{56-60}$ In this respect, our method bears some resemblance with the optimally tuned screened range separated hybrid functionals (OT-SRSH) approach ${ }^{61}$ coupled with a non-equilibrium PCM model, where the tuning of the amount of long-range Fock exchange is performed by using energies calculated with DFT+PCM. ${ }^{58-60,62}$

We then extend this approach to compute BSE spectra of molecules in solution using the full embedding QM/MM scheme, i.e $\mathrm{BSE} / G W_{e} / \mathrm{DFT}_{e} @ \operatorname{PBEh}\left(\alpha_{e}\right)$. In order to demonstrate the validity of the method, we compute the optical absorption spectra of $10 \mathrm{AB}$ derivatives shown in Fig. 5 that exhibit varying values of $\mathrm{S}_{1}$ band separation, $\Delta \mathrm{E}\left(\mathrm{S}_{1}\right)$, between trans and cis. For this purpose, we have chosen azo derivatives that exhibit (experimental) values of 
$\Delta \mathrm{E}\left(\mathrm{S}_{1}\right)$ ranging from $0.13 \mathrm{eV}$ for 1 to $0.54 \mathrm{eV}$ for 6 (see Table S7 and references within). All experimental values are taken from the absorption spectra as the maxima of the $n \rightarrow \pi^{*}$ transition $^{5,63-66}$ and they are tabulated in Table S7. To describe each isomer in solution we use the following procedure: (1) optimize the geometry using $\mathrm{DFT}_{e} @ \mathrm{PBE} 0$; (2) determine the $\alpha$ that minimized $J(\alpha)$, which requires few $G W_{e} / \mathrm{DFT}_{e} @ \mathrm{PBEh}$ calculations at different $\alpha,(3)$ compute the optical spectra using BSE at the optimal $\alpha$ (see fig. 1).

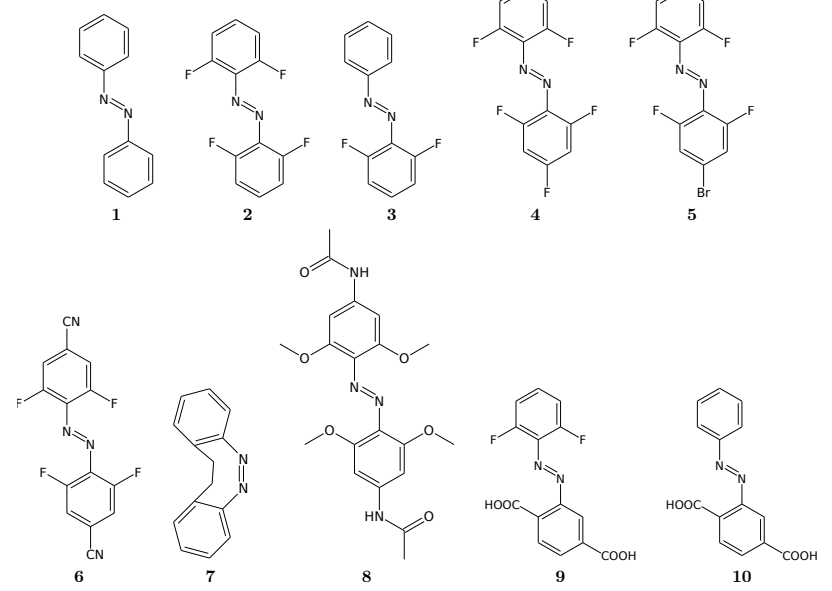

Figure 5. Schematic illustration of the $\mathbf{A B}$ derivatives studied in this work.
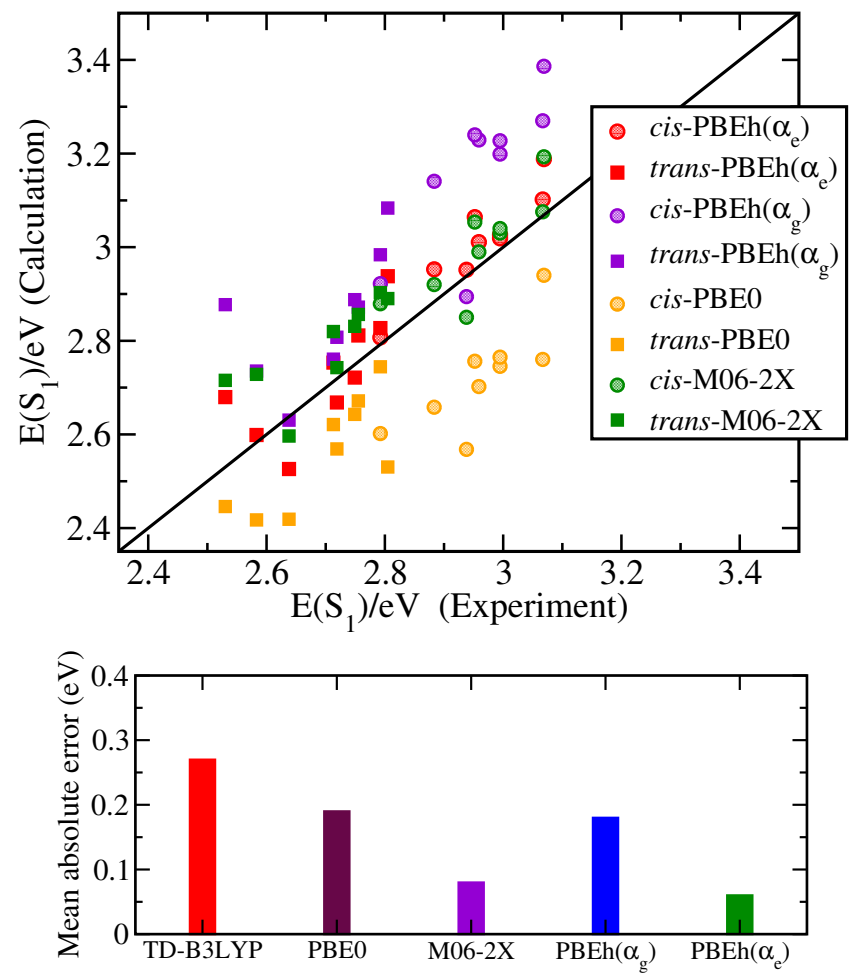

Figure 6. Experiment versus calculation comparison for the first excitation energy, $\mathrm{E}\left(\mathrm{S}_{1}\right)$, of the $\mathbf{A B}$ derivatives in the solvated phase and MAEs. The experimental values ${ }^{5,63-66}$ taken as reference are tabulated in Table $\mathrm{S} 2$. The full list of $\mathrm{E}\left(\mathrm{S}_{1}\right)$ is reported in Table S8. The MAE of TDDFT is taken from published data. ${ }^{63}$

For clarity, we report first the case of $\mathbf{1}$-cis and 2-cis, i.e. bare $\mathbf{A B}$ and ortho-fluoro-AB (see Fig. 5). In Fig. 4 we compare the BSE spectra computed using different levels of embedding with the experimental spectra measured in ace- tonitrile. Only a full embedding approach with the environment consistent IP choice (i.e. using $\alpha_{e}$ ) reproduces the optical spectra of 1-cis and 2-cis molecules (magenta curves) in acetonitrile with an accuracy of less than a tenth of $\mathrm{eV}$ for $\mathrm{E}\left(\mathrm{S}_{1}\right)$ (see Fig. 4), compared with experiment (black curves). Similar results are found for trans and are reported in Figs. S6 and S7. It is interesting to note that although one would expect to improve the agreement with experiment upon inclusion of embedding with $G W_{e} @ \mathrm{DFT}_{e}$ but using $\alpha_{g}$ (yellow curve in Fig. 4), the comparison instead worsens compared with the gas phase calculations (green curves). As for the the case of acrolein, when keeping the same starting $\mathrm{XCF}$, we observe a blueshift for the $n \rightarrow \pi^{*}$ excitation upon solvation. ${ }^{41}$ While for acrolein the blueshift resulted mainly from the effect of embedding at the DFT ground state level (Table S4), here embedding at $\mathrm{DFT}_{e}$ and $\mathrm{GW}_{e}$ contribute by similar amounts to the total shift as shown in Figs. S6 and S7. The wavefunction is also modified when the SMD model is used in DFT almost as much as upon changing $\alpha$, as illustrated in Figs. S10 and S11. To further assess the accuracy of this approach we report the value of and $\Delta \mathrm{E}\left(\mathrm{S}_{1}\right)$ for molecules $\mathbf{1}$ and $\mathbf{2}$ using 4 different computational schemes that gradually include the solvent effects to a full embedding scheme, and again, compare it with experiment. The $\mathrm{S}_{1}$ excitation energies reported in Table 1 show that the best agreement with experiment is obtained with $\mathrm{BSE} / G W_{e} / \mathrm{DFT}_{e} @ \operatorname{PBEh}\left(\alpha_{e}\right)$.

Table 1. $\mathrm{S}_{1}$ excitation energies and band separation, $\Delta \mathrm{E}\left(\mathrm{S}_{1}\right)$ in $\mathrm{eV}$, of 1-trans and cis and 2 computed using different embedding schemes. For clarity we use the notation $\operatorname{DFT}(\alpha)$ rather than DFT@PBEh $(\alpha)$.

\begin{tabular}{|c|c|c|c|c|c|c|c|}
\hline \multirow{2}{*}{ isomer } & \multirow[b]{2}{*}{$\alpha_{g}$} & \multirow[b]{2}{*}{$\alpha_{e}$} & \multicolumn{2}{|c|}{$\overline{\mathrm{BSE}} / G W_{g}$} & \multicolumn{2}{|c|}{$\overline{\mathrm{BSE}} / G W_{e}$} & \multirow[b]{2}{*}{ Exp. } \\
\hline & & & $\operatorname{DFT}_{\mathrm{g}}\left(\alpha_{g}\right)$ & $\operatorname{DFT}_{\mathrm{e}}\left(\alpha_{g}\right)$ & $\operatorname{DFT}_{\mathrm{e}}\left(\alpha_{g}\right)$ & $\operatorname{DFT}_{\mathrm{e}}\left(\alpha_{e}\right)$ & \\
\hline 1-trans & 0.63 & 0.48 & 2.835 & 2.896 & 3.002 & 2.893 & 2.804 \\
\hline 1-cis & 0.59 & 0.45 & 3.016 & 3.077 & 3.112 & 2.914 & 2.882 \\
\hline 1- $\Delta \mathrm{E}\left(\mathrm{S}_{1}\right)$ & - & - & 0.182 & 0.181 & 0.110 & 0.022 & 0.078 \\
\hline 2-trans & 0.62 & 0.44 & 2.529 & 2.707 & 2.816 & 2.668 & 2.719 \\
\hline 2-cis & 0.59 & 0.46 & 3.121 & 3.194 & 3.227 & 3.027 & 2.995 \\
\hline 2- $\Delta \mathrm{E}\left(\mathrm{S}_{1}\right)$ & - & - & 0.592 & 0.486 & 0.411 & 0.358 & 0.276 \\
\hline
\end{tabular}

Finally, the $\mathrm{BSE} / G W_{e} / \mathrm{DFT}_{e} @ \operatorname{PBEh}\left(\alpha_{e}\right)$ excitation energies of the 20 geometries (two isomers for each of the 10 derivatives) in different organic solvents are reported in Fig. 6 and compared with experimental values. It should be noted that the values of $\alpha_{e}$ are determined for each AB derivative and trans-cis configurations, and vary only slightly from case to case (from 0.35 for 5-trans to 0.46 for 3-cis, see Table S2 for the full list of values). An excellent agreement with experimentally observed first excitation energies is achieved for both trans and cis (see Fig. 6) with a mean absolute error (MAE) for $\mathrm{S}_{1}$ as low as $0.06 \mathrm{eV}$. For comparison, we also compute the BSE spectra using the full embedding scheme while using i) PBE0, ii) M06-2X, and iii) $\operatorname{PBEh}\left(\alpha_{g}\right)$ throughout. Thus, the calculations follow the scheme BSE $/ G W_{e} / \mathrm{DFT}_{e} @ \mathrm{PBE} 0, \mathrm{BSE} / G W_{e} / \mathrm{DFT}_{e} @ \mathrm{M} 06-$ $2 \mathrm{X}$ and $\mathrm{BSE} / G W_{e} / \mathrm{DFT}_{e} @ \operatorname{PBEh}\left(\alpha_{g}\right)$, respectively. We also include results from a previous study using TDDFT@B3LYP with molecules embedded in a continuum using PCM. ${ }^{63}$ All data points are shown in Fig. 6 together with the MAEs of each set of calculations. The whole set of $\mathrm{E}\left(\mathrm{S}_{1}\right)$ excitation energies are tabulated in Table S8. The MAE is slightly larger for M06-2X $(0.08 \mathrm{eV})$ while it increases by more than a factor of three for PBE0 $(0.19 \mathrm{eV})$. Interestingly, $\operatorname{PBEh}\left(\alpha_{g}\right)$ performs similarly to $\mathrm{PBE} 0$ with a MAE of 0.18 : while the former overestimates $\mathrm{E}\left(\mathrm{S}_{1}\right)$ in general, the latter underestimates it. This reveals again the importance of the good 
amount of exact exchange. Finally, our approach largely outperforms recent embedded TDDFT calculations ${ }^{63}$ with $\mathrm{MAE}=0.28 \mathrm{eV}$ (data shown in Fig. S12).

In conclusion, our calculations using the Bethe-Salpeter formalism combined with a non-equlibrium embedding scheme show an excellent agreement with experiments for the lowest singlet energy of a large set of solvated azobenzene derivatives. We evidence in particular the importance of tuning the starting point functional in systems where not only the energy levels, but also the shape of the KS molecular orbitals, may vary significantly as a function of the selected functional. This may lead to significant effects in the case of the very standard non-self-consistent or partially selfconsistent BSE/GW schemes where the input Kohn-Sham eigenstates are kept frozen. The alignment of the KS and $G W$ HOMO level energies offers a mean to select the starting functional on an ab initio basis, yielding an improved asymptotic density and leading, in the present case, to an excellent agreement with experimental data.

Acknowledgement This work was funded by the French National Agency for Research (ANR-15-CE06-0003-01). Calculations were performed using resources granted by GENCI under the CINES grant number A0020907211. The froggy platform of the CIMENT infrastructure was also employed for the calculations.

Supporting Information Available: Convergence studies; BSE of acrolein in water; excitation energies and oscillator strengths of 1-trans/cis computed with different ab initio methods; KS wavefunctions; BSE with different embedding schemes; tabulated $\mathrm{S}_{1}$ energies and $\alpha_{e}$; MEAs. This material is available free of charge via the Internet at http://pubs.acs.org/.

\section{Notes and References}

(1) Velema, W. A.; Van Der Berg, J. P.; Hansen, M. J.; Szymanski, W.; Driessen, A. J.; Feringa, B. L. Optical control of antibacterial activity. Nature chemistry 2013, 5, 924.

(2) Beharry, A. A.; Sadovski, O.; Woolley, G. A. Azobenzene photoswitching without ultraviolet light. J. Am. Chem. Soc. 2011 133, 19684-19687.

(3) Matsunaga, D.; Asanuma, H.; Komiyama, M. Photoregulation of RNA digestion by RNase $\mathrm{H}$ with Azobenzene-tethered DNA. J. Am. Chem. Soc. 2004, 126, 11452-11453.

(4) Wang, F.; Liu, X.; Willner, I. DNA Switches: from Principles to Applications. Angew. Chem. Int. Ed. 2015, 54, 1098-1129.

(5) Park, J.; Yuan, D.; Pham, K. T.; Li, J.-R.; Yakovenko, A.; Zhou, H.-C. Reversible alteration of $\mathrm{CO} 2$ adsorption upon photochemical or thermal treatment in a metal-organic framework. J. Am. Chem. Soc. 2011, 134, 99-102.

(6) Yang, C.-T.; Kshirsagar, A. R.; Eddin, A. C.; Lin, L.-C. Poloni, R. Tuning Gas Adsorption by Metal Node Blocking in Photoresponsive Metal-Organic Frameworks. Chem. Eur. J. 2018, 24, 15167-15172.

(7) Wang, Z.; Knebel, A.; Grosjean, S.; Wagner, D.; Bräse, S. Wöll, C.; Caro, J.; Heinke, L. Tunable Molecular Separation by Nanoporous Membranes. Nat. Commun. 2016, 7, 13872.

(8) Vetráková, L.; Ladányi, V.; Al Anshori, J.; Dvořák, P.; Wirz, J. Heger, D. The absorption spectrum of cis-azobenzene. Photochem. Photobiol. Sci. 2017, 16, 1749-1756.

(9) Zimmerman, G.; Chow, L.-Y.; Paik, U.-J. The Photochemical Isomerization of Azobenzene. J. Am. Chem. Soc. 1958, 80, 3528-3531.

(10) Bandara, H. M. D.; Burdette, S. C. Photoisomerization in Different Classes of Azobenzene. Chem. Soc. Rev. 2012, 41, 18091825 .

(11) Siewertsen, R.; Neumann, H.; Buchheim-Stehn, B.; Herges, R.; Näther, C.; Renth, F.; Temps, F. Highly Efficient Reversible Z-E Photoisomerization of a Bridged Azobenzene with Visible Light through Resolved S1 ( $\left.\mathrm{n} \pi^{*}\right)$ Absorption Bands. J. Am. Chem. Soc. 2009, 131, 15594-15595.

(12) Bleger, D.; Schwarz, J.; Brouwer, A. M.; Hecht, S. oFluoroazobenzenes as readily synthesized photoswitches offering nearly quantitative two-way isomerization with visible light. $J$. Am. Chem. Soc. 2012, 134, 20597-20600.

(13) Pal, A. K.; Duignan, T. J.; Autschbach, J. Calculation of Linear and Nonlinear Optical Properties of Azobenzene Derivatives with Kohn-Sham and Coupled-Cluster Methods. Phys. Chem. Chem. Phys. 2018, 20, 7303-7316.
(14) Perdew, J. P.; Levy, M. Comment on "Significance of the Highest Occupied Kohn-Sham Eigenvalue". Phys. Rev. B 1997, 56, 16021-16028.

(15) Perdew, J. P.; Parr, R. G.; Levy, M.; Balduz, J. L. DensityFunctional Theory for Fractional Particle Number: Derivative Discontinuities of the Energy. Phys. Rev. Lett. 1982, 49, 16911694.

(16) Almbladh, C. O.; Pedroza, A. C. Density-Functional ExchangeCorrelation Potentials and Orbital Eigenvalues for light atoms. Phys. Rev. A 1984, 29, 2322-2330.

(17) Casida, M. E. Correlated Optimized Effective-Potential Treatment of the Derivative Discontinuity and of the Highest Occupied Kohn-Sham Eigenvalue: A Janak-type Theorem for the Optimized Effective-Potential Model. Phys. Rev. B 1999, 59, 4694-4698.

(18) Chong, D. P.; Gritsenko, O. V.; Baerends, E. J. Interpretation of the Kohn-Sham Orbital Energies as Approximate Vertical Ionization Potentials. J. Chem. Phys. 2002, 116, 1760-1772.

(19) Strinati, G. Application of the Green's Functions Methods to the Study of the Optical Properties of Semiconductors. Riv. Nuovo Cim. 1988, 11, 1-86.

(20) Onida, G.; Reining, L.; Rubio, A. Electronic Excitations: Density-Functional versus Many-Body Green's-Function Approaches. Rev. Mod. Phys. 2002, 74, 601-659.

(21) Sottile, F.; Marsili, M.; Olevano, V.; Reining, L. Efficient Ab Initio Calculations of Bound and Continuum Excitons in the Absorption Spectra of Semiconductors and Insulators. Phys. Rev. B 2007, 76, 161103 .

(22) Jacquemin, D.; Duchemin, I.; Blase, X. Is the Bethe-Salpeter formalism accurate for excitation energies? Comparisons with TD-DFT, CASPT2, and EOM-CCSD. J. Phys. Chem. Lett. 2017, 8, 1524-1529.

(23) Bruneval, F.; Hamed, S. M.; Neaton, J. B. A Systematic Benchmark of the Ab Initio Bethe-Salpeter Equation Approach for Low-lying Optical Excitations of Small Organic Molecules. J. Chem. Phys. 2015, 142, 244101.

(24) Jacquemin, D.; Duchemin, I.; Blase, X. Benchmarking the Bethe-Salpeter Formalism on a Standard Organic Molecular Set. J. Chem. Theory Comput. 2015, 11, 3290-3304.

(25) Cembran, A.; Bernardi, F.; Garavelli, M.; Gagliardi, L.; Orlandi, G. On the Mechanism of the cis-trans Isomerization in the Lowest Electronic States of Azobenzene: S0, S1, and T1. J. Am. Chem. Soc. 2004, 126, 3234-3243.

(26) Conti, I.; Garavelli, M.; Orlandi, G. The Different Photoisomerization Efficiency of Azobenzene in the Lowest $n \pi *$ and $\pi \pi *$ Singlets: The Role of a Phantom State. J. Am. Chem. Soc. 2008, 130, 5216-5230.

(27) Fliegl, H.; Köhn, A.; Hättig, C.; Ahlrichs, R. Ab Initio Calculation of the Vibrational and Electronic Spectra of trans-and cis-Azobenzene. J. Am. Chem. Soc. 2003, 125, 9821-9827.

(28) Baumeier, B.; Andrienko, D.; Rohlfing, M. Frenkel and ChargeTransfer Excitations in Donor-acceptor Complexes from ManyBody Green's Functions Theory. J. Chem. Theory Comput. 2012, 8, 2790-2795.

(29) Faber, C.; Boulanger, P.; Attaccalite, C.; Duchemin, I.; Blase, X. Excited states properties of organic molecules: from density functional theory to the GW and Bethe-Salpeter Green's function formalisms. Phil. Trans. R. Soc. A 2014, 372 , 20130271

(30) Baumeier, B.; Rohlfing, M.; Andrienko, D. Electronic Excitations in Push-Pull Oligomers and Their Complexes with Fullerene from Many-Body Green's Functions Theory with Polarizable Embedding. J. Chem. Theory Comput. 2014, 10 3104-3110.

(31) Li, J.; D'Avino, G.; Duchemin, I.; Beljonne, D.; Blase, X. Combining the Many-Body GW Formalism with Classical Polarizable Models: Insights on the Electronic Structure of Molecular Solids. J. Phys. Chem. Lett. 2016, 7, 2814-2820.

(32) Duchemin, I.; Jacquemin, D.; Blase, X. Combining the GW formalism with the polarizable continuum model: A state-specific non-equilibrium approach. J. Chem. Phys. 2016, $144,164106$.

(33) Wehner, J.; Brombacher, L.; Brown, J.; Junghans, C.; Caylak, O.; Khalak, Y.; Madhikar, P.; Tirimbò, G.; Baumeier, B. Electronic Excitations in Complex Molecular Environments: Many-Body Green's Functions Theory in VOTCA-XTP. J. Chem. Theory Comput. 2018, 14, 6253-6268.

(34) Blase, X.; Attaccalite, C. Charge-Transfer Excitations in Molecular Donor-Acceptor Complexes within the Many-Body BetheSalpeter Approach. Appl. Phys. Lett. 2011, 99, 171909.

(35) Blase, X.; Attaccalite, C.; Olevano, V. First-Principles $G W$ calculations for fullerenes, porphyrins, phtalocyanine, and other Molecules of Interest for Organic Photovoltaic Applications. Phys. Rev. B 2011, 83, 115103.

(36) Valiev, M.; Bylaska, E.; Govind, N.; Kowalski, K. Straatsma, T.; Dam, H. V.; Wang, D.; Nieplocha, J.; Apra, E. Windus, T.; de Jong, W. NWChem: A Comprehensive and Scalable Open-Source Solution for Large Scale Molecular Simulations. Comput. Phys. Commun. 2010, 181, $1477-1489$.

(37) Kaplan, F.; Harding, M. E.; Seiler, C.; Weigend, F.; Evers, F.; van Setten, M. J. Quasi-Particle Self-Consistent GW for Molecules. J. Chem. Theory Comput. 2016, 12, 2528-2541.

(38) Marenich, A. V.; Cramer, C. J.; Truhlar, D. G. Universal Solvation Model based on Solute Electron Density and on a Continuum Model of the Solvent defined by the Bulk Dielectric Con- 
stant and Atomic Surface Tensions. J. Phys. Chem. B 2009 113, 6378-6396.

(39) Winget, P.; Dolney, D. M.; Giesen, D. J.; Cramer, C. J.; Truhlar, D. G. Minnesota solvent descriptor database. Dept. of Chemistry and Supercomputer Inst., University of Minnesota, Minneapolis, MN 1999, 55455.

(40) Li, J ; D'Avino, G.; Duchemin, I; Beljonne, D ; Blase, X. Accurate description of charged excitations in molecular solids from embedded many-body perturbation theory. Phys. Rev. B 2018, 97, 035108

(41) Duchemin, I.; Guido, C. A.; Jacquemin, D.; Blase, X. The Bethe-Salpeter formalism with polarisable continuum embedding: reconciling linear-response and state-specific features. Chem. Sci. 2018, 9, 4430-4443.

(42) D'Avino, G.; Muccioli, L.; Zannoni, C.; Beljonne, D.; Soos, Z. G. Electronic Polarization in Organic Crystals: A Comparative Study of Induced Dipoles and Intramolecular Charge Redistribution Schemes. J. Chem. Theory Comput. 2014, 10, 49594971.

(43) The same database is used within NWchem http://comp.chem.umn.edu/solvation/mnsddb.pdf.

(44) Adamo, C.; Barone, V. Toward reliable density functional methods without adjustable parameters: The PBE0 model. J. Chem. Phys. 1999, 110, 6158-6170.

(45) Dunning Jr, T. H. Gaussian Basis Sets for Use in Correlated Molecular Calculations. I. The Atoms Boron through Neon and Hydrogen. J. Chem. Phys. 1989, 90, 1007-1023.

(46) Woon, D. E.; Dunning Jr, T. H. Gaussian basis sets for use in correlated molecular calculations. III. The atoms aluminum through argon. J. Chem. Phys. 1993, 98, 1358-1371.

(47) Burke, K.; Ernzerhof, M.; Perdew, J. P. The Adiabatic Connection Method: a Non-empirical Hybrid. Chemical Physics Letters 1997, 265, 115-120.

(48) Perdew, J. P.; Burke, K.; Ernzerhof, M. Generalized Gradient Approximation Made Simple. Phys. Rev. Lett. 1996, 77, 38653868.

(49) Blase, X.; Duchemin, I.; Jacquemin, D. The Bethe-Salpeter Equation in Chemistry: Relations with TD-DFT, Applications and Challenges. Chem. Soc. Rev. 2018, 47, 1022-1043.

(50) Zhao, Y.; Truhlar, D. G. The M06 Suite of Density Functionals for Main Group Thermochemistry, Thermochemical Kinetics, Noncovalent Interactions, Excited States, and Transition Elements: Two New Functionals and Systematic Testing of Four M06-class Functionals and 12 other Functionals. Theor. Chem. Acc. 2008, 120, 215-241.

(51) Levy, M.; Perdew, J. P.; Sahni, V. Exact differential equation for the density and ionization energy of a many-particle system. Phys. Rev. A 1984, 30, 2745.

(52) Perdew, J. P.; Parr, R. G.; Levy, M.; Balduz Jr, J. L. Densityfunctional theory for fractional particle number: derivative discontinuities of the energy. Phys. Rev. Lett. 1982, 49, 1691.

(53) Perdew, J. P.; Levy, M. Comment on âĂIJSignificance of the highest occupied Kohn-Sham eigenvalueâĂİ. Phys. Rev. B 1997, 56, 16021 .

(54) Katriel, J.; Davidson, E. R. Asymptotic behavior of atomic and molecular wave functions. Proc. Natl. Acad. Sci. 1980, 7r, 4403-4406.

(55) Conti, I.; Garavelli, M.; Orlandi, G. The different photoisomerization efficiency of azobenzene in the lowest $\mathrm{n} \pi^{*}$ and $\pi \pi^{*} \sin$ glets: the role of a phantom state. J. Am. Chem. Soc. 2008 $130,5216-5230$

(56) Sachse, T.; Martínez, T. J.; Presselt, M. On combining the conductor-like screening model and optimally tuned rangeseparated hybrid density functionals. J. Chem. Phys. 2019 150,174117

(57) Kronik, L.; Kümmel, S. Dielectric screening meets optimally tuned density functionals. Adv. Mater. 2018, 30, 1706560.

(58) Bhandari, S.; Dunietz, B. D. Quantitative Accuracy in Calculating Charge Transfer State Energies in Solvated Molecular Complexes Using a Screened Range Separated Hybrid Functional within a Polarized Continuum Model. J. Chem. Theory Comput. 2019, 15, 4305-4311.

(59) Zheng, Z.; Egger, D. A.; Bredas, J.-L.; Kronik, L. Coropceanu, V. Effect of solid-state polarization on chargetransfer excitations and transport levels at organic interfaces from a screened range-separated hybrid functional. J. Phys. Chem. Lett. 2017, 8, 3277-3283.

(60) Bhandari, S.; Cheung, M. S.; Geva, E.; Kronik, L.; Dunietz, B. D. Fundamental Gaps of Condensed-Phase Organic Semiconductors from Single-Molecule Calculations using Polarization-Consistent Optimally Tuned Screened RangeSeparated Hybrid Functionals. J. Chem. Theory Comput. 2018, 14, 6287-6294.

(61) Refaely-Abramson, S.; Sharifzadeh, S.; Jain, M.; Baer, R.; Neaton, J. B.; Kronik, L. Gap renormalization of molecular crystals from density-functional theory. Phys. Rev. B 2013, 88, 081204 .

(62) Joo, B.; Han, H.; Kim, E.-G. Solvation-mediated tuning of the range-separated hybrid functional: Self-sufficiency through screened exchange. J. Chem. Theory Comput. 2018, 14, 28232828.

(63) Knie, C.; Utecht, M.; Zhao, F.; Kulla, H.; Kovalenko, S.;
Brouwer, A. M.; Saalfrank, P.; Hecht, S.; Bleger, D. orthoFluoroazobenzenes: Visible Light Switches with Very LongLived Z Isomers. Chem. Eur. J. 2014, 20, 16492-16501.

(64) Siewertsen, R.; Schönborn, J. B.; Hartke, B.; Renth, F. Temps, F. Superior $\mathrm{Z} \rightarrow \mathrm{E}$ and $\mathrm{E} \rightarrow \mathrm{Z}$ Photoswitching Dynamics of dihydrodibenzodiazocine, a Bridged Azobenzene, by $\mathrm{S}_{1}$ (n- $\pi *$ ) Excitation at $\lambda=387$ and $490 \mathrm{~nm}$. Phys. Chem. Chem. Phys. 2011, 13, 1054-1063.

(65) Beharry, A. A.; Sadovski, O.; Woolley, G. A. Azobenzene Photoswitching without Ultraviolet Light. J. Am. Chem. Soc. 2011 133, 19684-19687.

(66) Müller, K.; Knebel, A.; Zhao, F.; Bléger, D.; Caro, J.; Heinke, L. Switching Thin Films of Azobenzene-Containing Metal-Organic Frameworks with Visible Light. Chem. Eur. J. 2017, 23, 54345438 


\section{Graphical TOC Entry}

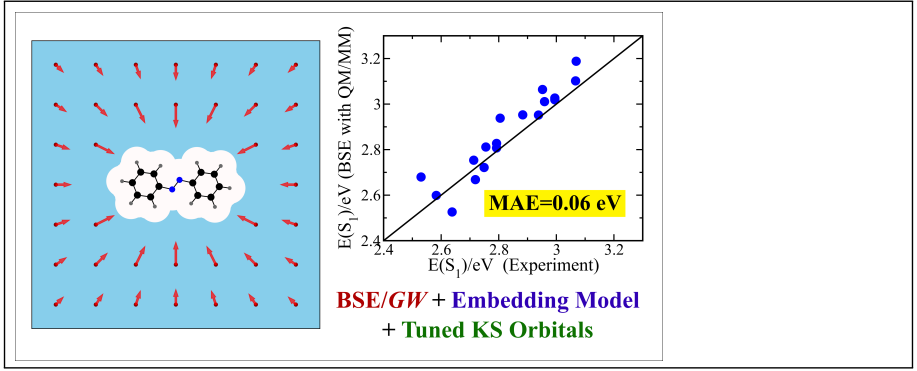

